$\begin{array}{cc}\text { ACADEMIA ROMÂNĂ } & \text { Rev. Roum. Chim., } \\ \text { Revis, 64(11), 993-998 } \\ \text { http://web.icf.ro/rrch/ } & \text { DOI: 10.33224/rrch.2019.64.11.08 }\end{array}$

\title{
A NEW REAGENT FOR EFFICIENT SYNTHESIS OF NITRILES FROM ALDOXIMES USING METHOXYMETHYL BROMIDE
}

\author{
Nesimi ULUDAG* and Ozge NUR GIDEN \\ Department of Chemistry, Faculty of Science and Arts, Tekirdag Namık Kemal University, \\ 59030 Tekirdag, Turkey
}

Received June 3, 2019

This study outlines an efficient, high-yielding, and rapid method by which to access diverse nitriles from aldoximes with methoxymethyl bromide (MOM-Br) in THF. It represents the first application of MOM-Br as a deoximation reagent to synthesize nitriles. The reaction was performed at reflux to ensure excellent yield (79-96\%) of the nitriles within 20-45 minutes. Furthermore, this method has been successfully applied to the synthesis of the synthesis precursor of aromatic, heteroaromatic, cyclic, and acyclic aliphatic.

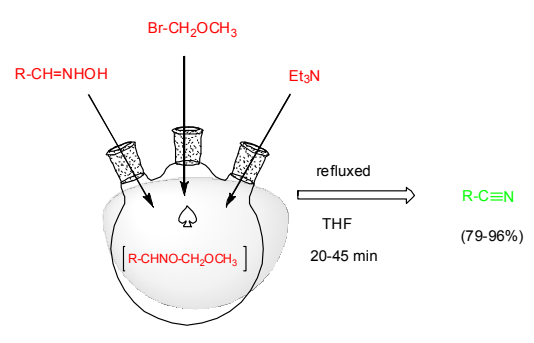

\section{INTRODUCTION}

The nitrile is an important structural motif in natural products, and it also serves as a pharmaceuticals product for a variety of medicinal indications. Several types of nitrile-containing compounds can be applied, ${ }^{1-3}$ such as vildagliptin (antidiabetic drug), letrozole (aromatase inhibitor), pericyazine (antipsychotic drug), and rilpivirine (anti-HIV agent) ${ }^{4,5}$ (See Fig. 1).

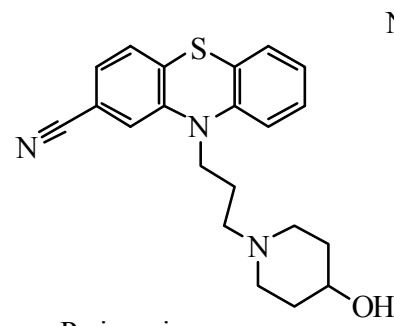

Pericyazine<smiles></smiles>

Letrozole

The derivatives of nitriles present great significance in chemistry and biology and are of great pharmacological interest. They have led to a sustained effort in the stimulated development of methodologies for their preparation. On the one hand, the nitrile unit is present in numerous compounds for natural products, agricultural chemicals, drugs, and dyes. On the other hand, the nitrile group Bosotinib serves as a valuable precursor in organic synthesis with a core of 3-cyanoquinoline; this group was recently approved by the Food and Drug Administration (FDA).

Vildagliptin

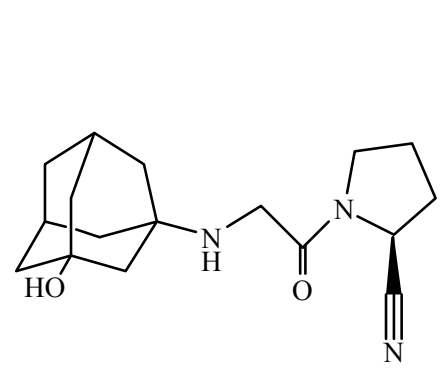<smiles>Cc1cc(/C=C/C#N)ccc1Nc1ccnc(Nc2ccc(C(=N)N)cc2)n1</smiles>

Rilpivirine

Fig. 1 - Examples of pharmaceutically significant nitrile containing molecules.

\footnotetext{
* Corresponding author: nuludag@nku.edu.tr
} 
Nitriles exhibit broad and varied applications and thus play a key role in organic chemistry., The preparation of nitriles using dehydration agents has been a very prominent means for the development of an anti-cancer drug. ${ }^{8}$ During recent decades, the use of various catalysts as a reagent has been established for the transformation of nitriles from substrates like oximes. ${ }^{7-15}$ Among the most popular approaches is, the conversion of aldoximes into nitriles via dehydration of intermediate aldoximes using expensive catalysts, various oxidants, prolonged reaction time, workup difficulties, hazardous metal salts, or various other existing methodologies. These approaches have been undesirable in terms of sustainable synthesis. ${ }^{16,17}$ Therefore, a simple conversion of aldoximes through dehydration to nitriles, preferably through the one-pot method, would be of significant value compared to the existing methods. Although various methods have been reported for the synthesis of nitriles, improvement of these methods is an interesting topic for the practice of organic chemistry. Thus, we have designed and realized various aldoximes into nitriles through the formation of oximes using methoxymethyl bromide in the presence of $\mathrm{Et}_{3} \mathrm{~N}$ and THF (Scheme 1). The tentatively proposed reaction mechanism is illustrated in Scheme 2.

Methoxymethyl bromide was initially introduced for use in the synthesis of aldoximes into nitriles, and it has also been used in other important organic reactions as a reagent. ${ }^{18,19}$

Our interest in nitrile chemistry has led us to consider the use of methoxymethyl bromide as an alternative to the efficient synthesis of nitriles from aldoximes under mild reaction conditions, i.e., in
THF under refluxed conditions. This method was applicable to the nitrile synthesis in complex molecules with various functions.

\section{RESULTS AND DISCUSSION}

It has been reported that aldoximes can be converted to nitriles using methoxymethyl bromide under refluxed condition (Scheme 1). Herein, we report the successful results of the dehydration of aldoximes to produce nitriles in the presence of $\mathrm{Et}_{3} \mathrm{~N}$ and THF.

The dehydration of aldoximes is of significant value compared to the existing methods. ${ }^{20,21}$ These catalysts provide a new and improved alternative to the existing methods in modern synthetic chemistry. Hence, during the course of these studies, we initially investigated the transformation of benzaldoxime to benzonitrile in the presence of tetrabutylammonium hydrogen sulfate, $25 \% \mathrm{NaOH}$ and methoxymethyl bromide in very low yield $(21 \%)$ and after a longer time period $(6 \mathrm{~h})$. Howover, in the presence of $\mathrm{Et}_{3} \mathrm{~N}$, THF, and methoxymethyl bromide, a high yield was achieved in a short time (Table 1). After the optimization of the reaction conditions, we used this method as a one-step procedure in a short and efficient synthesis of benzonitrile as a model substrate to establish the optimal reaction conditions. The results are shown in Table 1. Methoxymethyl bromide and tetrahydrofuran are also suitable as a reagent and solvent, respectively. However, tetrabutylammonium hydrogen sulfate and dichloromethane were inferior selections, and the reaction did not proceed cleanly using substances.

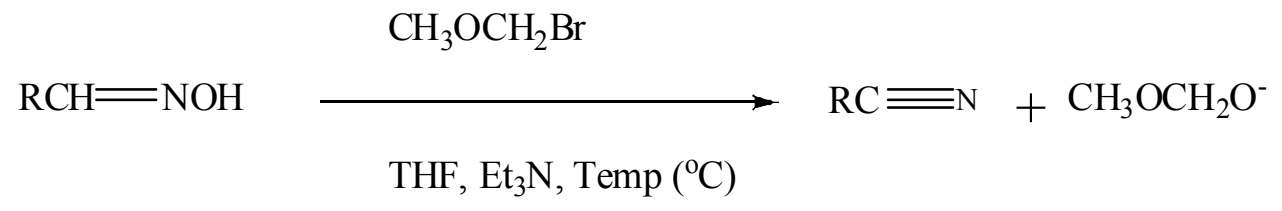

Scheme 1 - Dehydration of aldoximes to nitriles.

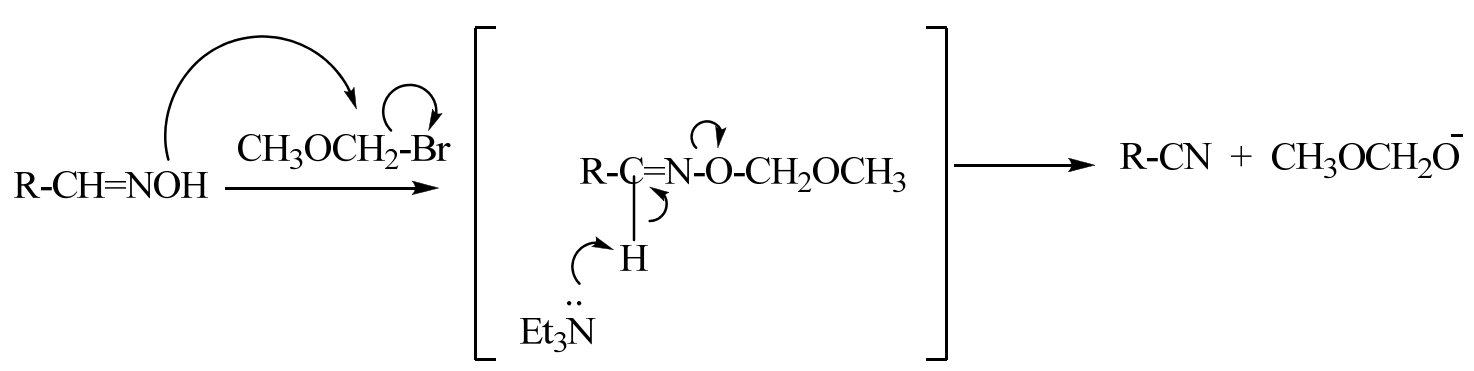

Scheme 2 - Possible mechanistic pathway for the dehydration of aldoximes. 
Table 1

Efficient conversion of aldoximes to nitriles using methoxymethyl bromide

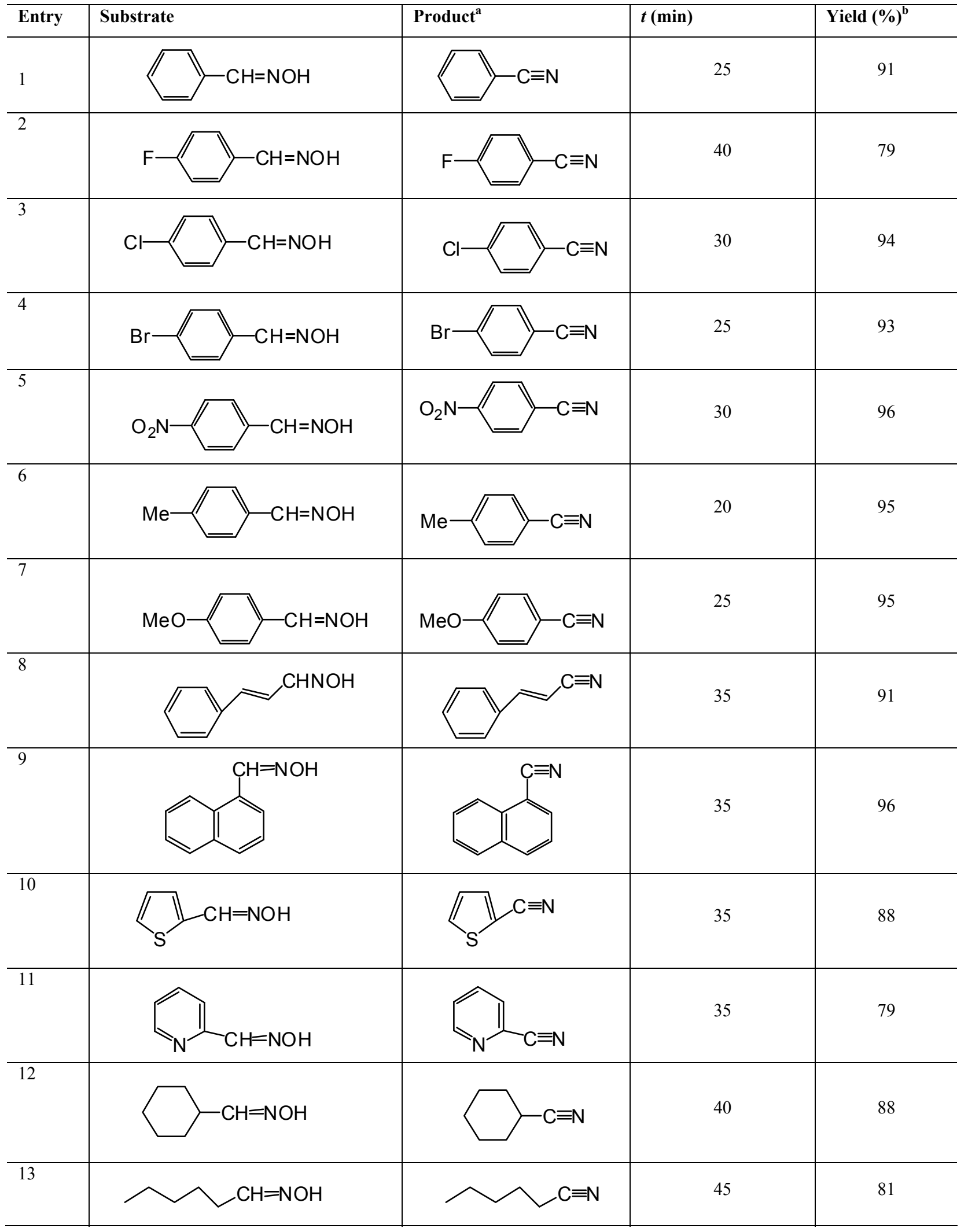

${ }^{\text {a }}$ Products were characterized by their melting points, IR, GC-MS, and NMR spectra.

${ }^{\mathrm{b}}$ The yields were determined after chromatographic purification (isolated yields). 
At this point, we assessed that the generalization of this transformation was subject to a variety of aldoximes as the starting material, which has been synthesized previously. ${ }^{22-27}$

Along this line of thought, we report the use of methoxymethyl bromide and $\mathrm{Et}_{3} \mathrm{~N}$ under the refluxed condition for the direct conversion of aldoximes into nitriles. After reaching satisfactory results of the refluxed condition in a short time (Table 1), we subsequently investigated the substrate scope of an aldoxime conversion to a nitrile. Both electron-withdrawing and electrondonating substituted aldoximes showed high yields under these conditions (Table 1, entry 1-7). Under the same conditions, cinnamaldoxime, heteroatomaldoximes, 2-naphthaldehyde oxime and cylicnoncylcic aldoximes were converted into nitriles with good yield (Table 1, entry 8-13). As shown in Table 1 , the reaction with methoxymethyl bromide proceeded smoothly. This makes the reaction more useful for the development of nitriles chemistry. Application of methoxymethyl bromide in the present cases also resulted in satisfactory conversion to niriles from a mechanistic consideration. The dehydration reaction process of the aldoximes with methoxymethyl bromide and $\mathrm{Et}_{3} \mathrm{~N}$ has also been evaluated, as shown in Scheme 2.

This result could be employed not only for the efficient conversion of aromatic aldoximes to aromatic cyano compounds, ${ }^{28}$ but also for aliphatic, cyclic, and heteroatom aldoximes. The present method can be readily applied to large scale processes with high efficiency and economically, and as a new catalyst system for dehydration of aldoximes. $^{29}$

\section{EXPERIMENTAL}

IR spectra were recorded by using a Mattson FT-IR spectrometer. ${ }^{1} \mathrm{H}-\mathrm{NMR}$ spectra were recorded on a Bruker 400 spectrometer at $400 \mathrm{MHz}$. Spectra were registered in $\mathrm{CDCl}_{3}$ using a solvent as internal standard at $400 \mathrm{MHz}$ for ${ }^{1} \mathrm{H}$ and ${ }^{13} \mathrm{C}$ at $25{ }^{\circ} \mathrm{C}$. Melting points were taken in a capillary tube on Electro thermal IA 9000 apparatus and were uncorrected. Mass spectra were measured on a Micromass UK Platform II GC-MS spectrometer and HP 5971 mass and combined 5980 gas chromatography system. Combustion analysis of compounds was obtained on a CHNS-932-LECO. Column chromatography was performed using 60-120 mesh silica gel and reactions were monitored by thin layer chromatography (silica gel 60 F254) and aluminum oxide 90 active neutral. All chemicals and solvents were purchased from commercial sources. Purifications of the solvents were performed according to the standard methods

\section{General procedure for the preparation of Nitriles (Entry 1-13)}

For the preparation of nitrile from aldoxime using a methoxymethyl bromide as the dehydration agent, $\mathrm{Et}_{3} \mathrm{~N}$ (1.5 mmol) was added to a stirred mixture of aldoxime $(1.0 \mathrm{mmol})$ and $10 \mathrm{~mL}$ of THF, followed by methoxymethyl bromide $(1.5 \mathrm{mmol})$. The resulting mixture was refluxed for a specified period (Table 1). The reaction was monitored by TLC (ethyl acetate: petroleum ether, 1:1). After completion of reaction, the contents were poured into water and neutralized with $\mathrm{NaHCO}_{3}$ solution $(20 \mathrm{~mL}, 10 \%)$, and then extracted with ethylacetate $(10 \mathrm{~mL} \times 3)$. The combined organic mixture was dried over anhydrous $\mathrm{Na}_{2} \mathrm{SO}_{4}$, concentrated and the residue was purified by column chromatography on silica gel (60-120 mesh) using ethyl acetate, and then the solvent was removed at reduced pressure to give products in 79\%-96\% yields (Entry 1-13). The nitriles characterized by LC-MS, ${ }^{1} \mathrm{H}$ NMR and ${ }^{13} \mathrm{C}$ NMR analysis.

Benzonitrile (Entry 1) Colorless oil $;^{30}$ yield (91\%); $R_{f}$ (EtOAc): 0.63; IR (KBr): $2220(\mathrm{CN}) \mathrm{cm}^{-1} ;{ }^{1} \mathrm{H}$ NMR (400 MHz, $\left.\mathrm{CDCl}_{3}\right): \delta$ 7.67-7.65 $(\mathrm{m}, 2 \mathrm{H}), 7.65-7.57(\mathrm{~m}, 1 \mathrm{H}), 7.49-7.44$ $(\mathrm{m}, 2 \mathrm{H}) ;{ }^{13} \mathrm{C}$ NMR $\left(100 \mathrm{MHz}, \mathrm{CDCl}_{3}\right): \delta 132.8,132.2,129.2$,

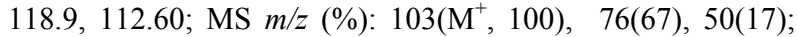
Anal. Calcd. For $\mathrm{C}_{7} \mathrm{H}_{5} \mathrm{~N}$ : C 81.53; $\mathrm{H} 4.89 ; \mathrm{N} 13.58$; Found : $\mathrm{C}$ 81.66; H 4.77; N $13.63 \%$.

4-Fluorobenzonitrile (Entry 2) Yellow oil; ${ }^{21}$ yield (79\%); $R_{f}$ (EtOAc): 0.68; IR (KBr): $2224(\mathrm{CN}) \mathrm{cm}^{-1} ;{ }^{1} \mathrm{H}$ NMR $(400$ $\left.\mathrm{MHz}, \mathrm{CDCl}_{3}\right): \delta$ 7.71-7.67 (m, 2H), 7.21-7.17 (m, 2H). ${ }^{13} \mathrm{C}$ NMR $\left(100 \mathrm{MHz}, \mathrm{CDCl}_{3}\right): \delta 166.3,134.7,118.0,116.9,108.5$; MS $m / z(\%): 122\left(\mathrm{M}^{+},+1\right)(7), 121$ (100), 93(47); Anal. Calcd. For $\mathrm{C}_{7} \mathrm{H}_{4} \mathrm{FN}$ : C 69.42; H 3.33; N 11.57; Found : C 69.54; H 3.26 ; N $11.65 \%$.

4-Chlorobenzonitrile (Entry 3) White solid; m.p. 92-93 ${ }^{0} \mathrm{C}$ (Lit. 93-94 $\left.{ }^{0} \mathrm{C}\right) ;{ }^{32}$ yield $(94 \%) ; \quad R_{f}$ (EtOAc): 0.73; IR ( $\left.\mathrm{KBr}\right)$ : $221(\mathrm{CN}) \mathrm{cm}^{-1} ;{ }^{1} \mathrm{H}$ NMR $\left(400 \mathrm{MHz}, \mathrm{CDCl}_{3}\right): \delta 7.60(\mathrm{~d}, J=7.1$ $\mathrm{Hz}, 2 \mathrm{H}), 7.33$ (d, $J=8.7 \mathrm{~Hz}, 2 \mathrm{H}) ;{ }^{13} \mathrm{C}$ NMR $(100 \mathrm{MHz}$, $\left.\mathrm{CDCl}_{3}\right): \delta 139.5,133.3,129.6,117.9,110.7 . \mathrm{MS} m / z(\%): \mathrm{MS}$ $m / z(\%): 138\left(\mathrm{M}^{+},+1\right)(65), 137$ (77), 101 (100), 74 (52). Anal. Calcd. For $\mathrm{C}_{7} \mathrm{H}_{4} \mathrm{ClN}$ : C 61.12; $\mathrm{H} 2.93$; N 10.18; Found : C 61.09; H 2.88; N 10.09\%.

4-Bromobenzonitrile (Entry 4) White solid; m.p. $111-112{ }^{0} \mathrm{C}$ (Lit. $\left.113-114{ }^{0} \mathrm{C}\right) ;{ }^{31}$ yield (93\%); $R_{f}$ (EtOAc): 0.55; IR (KBr): 2234(CN) $\mathrm{cm}^{-1} ;{ }^{1} \mathrm{H}$ NMR $\left(400 \mathrm{MHz}, \mathrm{CDCl}_{3}\right): \delta 7.63(\mathrm{~d}$, $J=8.3 \mathrm{~Hz}, 2 \mathrm{H}), 7.53(\mathrm{~d}, J=8.1 \mathrm{~Hz}, 2 \mathrm{H}) .{ }^{13} \mathrm{C}$ NMR $(100$ $\left.\mathrm{MHz}, \mathrm{CDCl}_{3}\right): \delta 133.4,132.6,128.0,118.0,111.2$. MS $\mathrm{m} / \mathrm{z}$ (\%): 183 (8), 182 (87), $181\left(\mathrm{M}^{+}\right)$(78), 101 (100), 75 ( 51). Anal. Calcd. For $\mathrm{C}_{7} \mathrm{H}_{4}$ BrN: C 46.19; H 2.22; N 7.70; Found : C 46.27 ; H 2.29; N 8.81\%.

4-Nitrobenzonitrile (Entry 5) White solid; m.p. $141-143{ }^{0} \mathrm{C}$ (Lit. $\left.141-145{ }^{0} \mathrm{C}\right) ;{ }^{33} R_{f}$ (EtOAc): 0.51 ; yield $(96 \%)$; IR (KBr): $2236(\mathrm{CN}) \mathrm{cm}^{-1}$; ${ }^{1} \mathrm{H}$ NMR $\left(400 \mathrm{MHz}, \mathrm{CDCl}_{3}\right): \delta 8.34(\mathrm{~d}$, $J=9.0 \mathrm{~Hz}, 2 \mathrm{H}), 7.93(\mathrm{~d}, J=9.0 \mathrm{~Hz}, 2 \mathrm{H}) ;{ }^{13} \mathrm{C} \mathrm{NMR}$ $\left(100 \mathrm{MHz}, \mathrm{CDCl}_{3}\right): \delta 149.9,133.4,124.2,118.2 .3,116.7$; MS $\mathrm{m} / \mathrm{z}(\%): 143\left(\mathrm{M}^{+}\right)$(58), 101 (100), 74 (61); MS m/z (\%): 148 $\left(\mathrm{M}^{+}, 45\right), 102$ (100), 74 (48); Anal. Calcd. For $\mathrm{C}_{7} \mathrm{H}_{4} \mathrm{~N}_{2} \mathrm{O}_{2}$ : C 56.76; H 2.72; N 18.91; Found : C 57.66; H 2.84; N 18.98\%.

4-Methybenzonitrile (Entry 6) Colorless oil; yield (95\%); ${ }^{31} R_{f}$ (EtOAc): 0.68; IR (KBr ): $2225(\mathrm{CN}) \mathrm{cm}^{-1}$; ${ }^{1} \mathrm{H}$ NMR $(400 \mathrm{MHz}$, $\left.\mathrm{CDCl}_{3}\right): \delta 7.52(\mathrm{~d}, J=8.2 \mathrm{~Hz}, 2 \mathrm{H}) \quad 7.26(\mathrm{~d}, J=7.8 \mathrm{~Hz}, 2 \mathrm{H})$, $2.44(\mathrm{~s}, 3 \mathrm{H}) ;{ }^{13} \mathrm{C}$ NMR $\left(100 \mathrm{MHz}, \mathrm{CDCl}_{3}\right): \delta 143.6,131.9$, 129.8, 119.1, 109.2, 21.8; MS $m / z(\%): 117\left(\mathrm{M}^{+}, 100\right), 90$ 
(44); Anal. Calcd. For $\mathrm{C}_{8} \mathrm{H}_{7} \mathrm{~N}$ : C 82.02; $\mathrm{H}$ 6.02; N 11.96; Found: C 81.82; H 6.18; N $11.88 \%$.

4-Methoxybenzonitrile (Entry 7) Colorless solid; m.p. 58$60{ }^{0} \mathrm{C}$ (Lit. $\left.58-61{ }^{0} \mathrm{C}\right){ }^{30,34} R_{f}$ (EtOAc): 0.57 ; yield (95\%); IR $(\mathrm{KBr}): 2218(\mathrm{CN}) \mathrm{cm}^{-1} ;{ }^{1} \mathrm{H}$ NMR $\left(400 \mathrm{MHz}, \mathrm{CDCl}_{3}\right): \delta 7.58$ $(\mathrm{d}, J=8.4 \mathrm{~Hz}, 2 \mathrm{H}), 6.94(\mathrm{~d}, J=8.4 \mathrm{~Hz}, 2 \mathrm{H}), 3.81(\mathrm{~S}, 3 \mathrm{H})$; ${ }^{13} \mathrm{C}$ NMR $\left(100 \mathrm{MHz}, \mathrm{CDCl}_{3}\right): \delta 162.9,134.1,119.3,114.8$, 104.1, 55.6; MS m/z (\%): $133\left(\mathrm{M}^{+}, 100\right), 102$ (59), 90 (68); Anal. Calcd. For $\mathrm{C}_{8} \mathrm{H}_{7} \mathrm{NO}$ : C 72.16; H 5.30; N 10.52; Found : C 72.23; H 5.24; N 10.59\%.

Cinnamonitrile (Entry 8) Colorless oil; yield (91\%); ${ }^{30,35} R_{f}$ (EtOAc):0.73; IR (KBr):2243 (CN) cm ${ }^{-1}$; ${ }^{1} \mathrm{H}$ NMR (400 MHz, $\left.\mathrm{CDCl}_{3}\right): \delta$ 7.47-7.36 $(\mathrm{m}, 6 \mathrm{H}$, aromatic $\mathrm{C}-\mathrm{H}$ and $\mathrm{PhCH}-\mathrm{H})$, $5.87(\mathrm{~d}, J=16.9 \mathrm{~Hz},-\mathrm{CH}-\mathrm{CN}) ;{ }^{13} \mathrm{C}$ NMR $\left(100 \mathrm{MHz}, \mathrm{CDCl}_{3}\right)$ : $\delta 150.6,133.6,131.3,129.2,127.4,118.2,96.4$; MS $m / z(\%)$ : $129\left(\mathrm{M}^{+}, 100\right), 102$ (48); Anal. Calcd. For $\mathrm{C}_{9} \mathrm{H}_{7} \mathrm{~N}$ : C 83.69; H 5.46; N 10.84; Found : C 83.54; H 5.38; N 10.89\%.

1-Naphthonitrile (Entry 9) Colorless oil; yield (96\%); ${ }^{31} R_{f}$ (EtOAc): 0.47; IR (KBr): $2223(\mathrm{CN}) \mathrm{cm}^{-1} ;{ }^{1} \mathrm{H}$ NMR $(400 \mathrm{MHz}$, $\left.\mathrm{CDCl}_{3}\right) \delta 8.22(\mathrm{~d}, J=8.3 \mathrm{~Hz}, 1 \mathrm{H}), \delta 8.04(\mathrm{~d}, J=8.3 \mathrm{~Hz}, 1 \mathrm{H})$, $\delta 7.69(\mathrm{t}, J=7.2 \mathrm{~Hz}, 2 \mathrm{H}), \delta 7.63(\mathrm{t}, J=7.4 \mathrm{~Hz}, 1 \mathrm{H}), \delta 7.61(\mathrm{t}$, $J=7.5 \mathrm{~Hz}, 1 \mathrm{H}), \delta 7.52(\mathrm{t}, J=7.2 \mathrm{~Hz}, 1 \mathrm{H}) ;{ }^{13} \mathrm{C} \mathrm{NMR}$ $\left(101 \mathrm{MHz}, \mathrm{CDCl}_{3}\right) \delta 133.2,132.8,132.5,132.2,128.4,128.7$, 127.4, 125.2, 124.7, 117.5, 110.3. MS $m / z(\%): 153\left(\mathrm{M}^{+}, 100\right)$, 126(17). Anal. Calcd. For $\mathrm{C}_{11} \mathrm{H}_{7} \mathrm{~N}$ : C 86.25; H 4.61; N 9.14; Found : C 86.18; H 4.69; N $9.08 \%$.

Thiophene-2-carbonitrile (Entry 10) Colorless Oil; yield $(88 \%) ;{ }^{30,36} R_{f}$ (EtOAc): 0.52; IR (KBr): $2288(\mathrm{CN}) \mathrm{cm}^{-1} ;{ }^{1} \mathrm{H}$ NMR $\left(400 \mathrm{MHz}, \mathrm{CDCl}_{3}\right): \delta 7.64-7.63(\mathrm{~m}, 1 \mathrm{H}), 7.60(\mathrm{dd}$, $J=5.3,1.4 \mathrm{~Hz}, 1 \mathrm{H}), 7.15(\mathrm{dd}, J=5.2,1.7 \mathrm{~Hz}) ;{ }^{13} \mathrm{C}$ NMR $(100$ $\left.\mathrm{MHz}, \mathrm{CDCl}_{3}\right): \delta 136.4,132.6,127.8,114.3,109.9 ; \mathrm{MS} \mathrm{m} / \mathrm{z}$ (\%): $109\left(\mathrm{M}^{+}, 100\right)$; Anal. Calcd. For $\mathrm{C}_{5} \mathrm{H}_{3} \mathrm{NS}$ : C 52.02; $\mathrm{H}$ 2.77; N 12.83; Found : C 52.11; H 2.69; N $12.89 \%$.

Picolinonitrile (Entry 11) Colorless oil; yield (79\%); ${ }^{37} R_{f}$ (EtOAc): 0.66; IR (KBr): $2234(\mathrm{CN}) \mathrm{cm}^{-1}$; ${ }^{1} \mathrm{H}$ NMR (400 $\left.\mathrm{MHz}, \mathrm{CDCl}_{3}\right): \delta 8.71(\mathrm{~s}, 1 \mathrm{H}), 7.88(\mathrm{~s}, 1 \mathrm{H}), 7.74(\mathrm{~s}, 1 \mathrm{H}), 7.61$ $(\mathrm{s}, 1 \mathrm{H}) ;{ }^{13} \mathrm{C}$ NMR $\left(100 \mathrm{MHz}, \mathrm{CDCl}_{3}\right): \delta 150.9,137.4,133.6$, 128.7, 127.3, 117.4; MS m/z (\%): $104\left(\mathrm{M}^{+}, 100\right), 77$ (48), 51 (28); Anal. Calcd. For $\mathrm{C}_{6} \mathrm{H}_{4} \mathrm{~N}_{2}$ : C 69.22; H 3.87; N 26.91; Found: C 69.14; H 3.77; N $26.82 \%$.

Cyclohexanacarbonitrile (Entry 12) Colorless oil; yield $(88 \%) ;{ }^{38} R_{f}$ (EtOAc): 0.68; FTIR (KBr): $2238(\mathrm{CN}) \mathrm{cm}^{-1} ;{ }^{1} \mathrm{H}$ NMR $\left(400 \mathrm{MHz}, \mathrm{CDCl}_{3}\right): \delta 2.59(\mathrm{tt}, J=8.3,3.8 \mathrm{~Hz}, 1 \mathrm{H})$, $1.84-1.80(\mathrm{~m}, 2 \mathrm{H}), 1.74-1.66(\mathrm{~m}, 4 \mathrm{H}), 1.46-1.35(\mathrm{~m}, 4 \mathrm{H}) ;{ }^{13} \mathrm{C}$ NMR (100 MHz, $\left.\mathrm{CDCl}_{3}\right): \delta 123.7,29.3,28.4,25.2,24.1$; MS m/z (\%): $109\left(\mathrm{M}^{+}, 100\right)$; Anal. Calcd. For $\mathrm{C}_{7} \mathrm{H}_{11} \mathrm{~N}$ : C 77.01; H 10.16; N 12.83; Found : C 76.87; H 10.09; N 12.92\%.

Hexanacarbonitrile (Entry 13) Colorless oil; yield (81\%); ${ }^{39}$ $R_{f}$ (EtOAc):0.47; IR (KBr, cm $\left.{ }^{-1}\right): 2241(\mathrm{CN}) ;{ }^{1} \mathrm{H}$ NMR $(400$ $\mathrm{MHz}, \mathrm{CDCl}_{3}$ ): $\delta 2.35$ (t, $\left.J=7.3 \mathrm{~Hz}, 2 \mathrm{H}\right), 1.66-1.41(\mathrm{~m}, 2 \mathrm{H})$, $1.40-1.36(\mathrm{~m}, 4 \mathrm{H}), 0.89(\mathrm{t}, J=7.2 \mathrm{~Hz}, 3 \mathrm{H}) ;{ }^{13} \mathrm{C} \mathrm{NMR}(100$ $\left.\mathrm{MHz}, \mathrm{CDCl}_{3}\right): \delta 121.130 .8,25.6,21.9,17.1,13.8 ; \mathrm{MS} \mathrm{m} / \mathrm{z}$ (\%): $97\left(\mathrm{M}^{+}, 100\right)$; Anal. Calcd. For $\mathrm{C}_{6} \mathrm{H}_{11} \mathrm{~N}$ : C 74.17; H 11.41; N 14.42; Found : C 74.23; H 11.34; N $14.55 \%$.

\section{CONCLUSION}

In summary, we utilized methoxymethyl bromide (MOM-Br) for the synthesis of nitriles through the dehydration of aldoximes. The compatibility of this method with the base offers rapid access to nitriles. Such a reaction can be produced simply under mild conditions and offers significant advantages over the existing methods in terms of high yields, an easily handled reagent system, and wide applicability among various substrates. The present study widens the scope of the utilization of numerous natural products and bioactive molecules in organic synthesis.

Acknowledgements. This work was supported by Tekirdag Namık Kemal University and this research also is part of Ozge Nur Giden's MSc thesis.

\section{REFERENCES}

1. F. F. Fleming, Nat. Prod. Rep., 1999, 16, 597-606.

2. J. S. Miller and J. L. Manson, Acc. Chem. Res., 2001, 34, 563-570.

3. H. He, P. Tran, H. Yin, H. Smith, Y. Batard, L. Wang, H. Einolf, H. Gu, J. B. Mangold, V. Fischer and D. Howard, Drug. Metab. Dispos., 2009, 37, 536-544.

4. M. Romero, P. Renard, D. H. Caignard, G. Atassi, X. Solans, P. Constants, C. Bailly and M. D. Pujol, J. Med. Chem., 2007, 50, 294-307, and references cited therein.

5. D. T. Mowry, Chem. Rev., 1948, 42, 189-283.

6. C. Bolm, J. Legros, J. Le Paih and L Zani, Chem. Rev., 2004, 104, 6217-6254.

7. C. M. Al Materneh, M. O. Apostu, I. I. Mangalagiu and R. Danac, Tetrahedron, 2016, 72, 4230-4238.

8. S. Fatahala, E. A. Shalaby, S. E. Kassab and M. S. Mohamed, Anti- Cancer Agent Med. Chem., 2015, 15, 517-526.

9. P. Ghos, B. Saha, G. C. Priyar, A. Tamang and R. Subba, Tetrahedron Lett., 2016, 57, 3618-3621.

10. K. Lee, S-B. Han, E-B, Yoo, S.-R. Chung, H. Oh and S. Hong, Synth. Commun., 2004, 34, 1775-1782.

11. Y. Song, D. Shen, Q. Zhang, B. Chen and G. Xu, Tetrahedron Lett., 2014, 55, 639-641.

12. A. V. Narsaiah, D. Sreenu and K. Nagaiah, Synth. Commun., 2006, 36, 137-140.

13. M. N. S. Rad, A. Khalafi-Nezhad, S. Behrouz, Z. Amini and M. Behrouz, Synth. Commun., 2010, 40, 2429-2440.

14. S. K. Dewan and R. Sing, Synth. Commun., 2003, 33, 3085-3088.

15. W. Jang, S. E. Kim, C. M. Yang, S. Yoon, M. Park, J. Lee, Y. Kim and M. Kim, Catal. Commun., 2015, 60, 120123.

16. G. A. Olah and D. Vanakar, Synthesis, 1978, 702-703.

17. G. A. Olah, D. Vanakar and A. L. Berrier, Synthesis, 1980, 45-46.

18. N. Pandurangan, Let. Org. Chem., 2017, 14, 231-235.

19. K. T. Reddy, R. Sreenivasulu and I. Hatti, Curr. Org. Chem., 2015, 12, 67-70.

20. A. Hulkenberg and J. J. Troost, Tetrahedron Lett., 1982, 23, 1505-1508.

21. F. Camps, V. Gasol and A. Guerrero, Synth. Commun., 1988, $18,445-452$.

22. N. D. Kokare and D. B. Shinde, Monatsh. Chem., 2009, 140, 185-188. 
23. K. Tambara and G. Pantos Dan, Org. Biomol. Chem., 2013, 11, 2466-2472.

24. M. Ramanat, S.-T. Liu, Tetrahedron, 2017, 73, 43174322.

25. W.-S. Wang, P. Chen, Y. Tang, Tetrahedron, 2017, 73, 2731-2739.

26. A. Primo, M. Puche, O. D. Pavel, B. Cojocaru, A. Tirsoaga, V. Parvulesco and V. Garcia, Chem. Commun., 2016, 52, 1839-1842.

27. C. V. Maftei, E. Fodor, P. G. Jones, C. G. Daniliuc, M. H. Franz, G. Kelter, H. H. Fiebig, M. Tamm, I. Neda, Rev. Roum. Chim., 2015, 60, 75-83.

28. N. Uludag and M. Yakup, Org. Prep. Proc. Int., 2015, 47, 454-460.

29. Ding R. Liu Y. Han M, W. Jiao, J. Li, H. Tian and B. Sun, J. Org. Chem., 2018, 83, 12939-12944.

30. S. A. Shipilovskikh, V. Y. Vaganov, E. I. Denisova, A. E. Rubtsov and A. V. Malkov, Org. Let., 2018, 20, 728-731.
31. L. Yu, H. Li, J. Ye, J. Liu, Q. Xu and M. Lautens, Org. Lett., 2014, 16, 1346-1349.

32. M. Hatsuda and M. Seki, Tetrahedron, 2005, 61, 99089917.

33. P. F. Fors and S. L. Buchwald, J. Am. Chem. Soc., 2009, 131, 12898-12899.

34. S. Kano, Y. Tanaka, E. Sugino, S. Shibuya and S. Hibino, Synthesis, 1980, 9, 741-742.

35. D. Saha, A. Saha and B. C. Ranu, Tetrahedron Lett., 2009, 50, 6088-6091.

36. H. C. Aspinall, O. Beckingham, M. D. Farrar, N. Greeves and C. D. Thomas, Tetrahedron Lett., 2011, 5, 51205123 .

37. C. Yang and J. M. Williams, Org. Lett., 2004, 6, 28372840.

38. S. Minakata, S. Okumura, T. Nagamachi and Y. Takeda, Org. Lett., 2011, 13, 2966-2969.

39. J. A. Campbell, G. McDouglad, H. McNab, L. V. Rees and R. G. Tayas, Synthesis, 2007, 20, 3179-3184. 